\title{
WAVES AND FLUCTUATIONS IN THE JOVIAN MAGNETOSPHERE
}

\author{
C. T. Russell ${ }^{1.2}$, D. E. Huddleston ${ }^{1}$, K. K. Khurana ${ }^{1}$ and M. G. Kivelson ${ }^{1,2}$ \\ ${ }^{1}$ Institute of Geophysics and Planetary Physics, University of California, \\ Los Angeles, CA 90095-1567, USA \\ ${ }^{2}$ Department of Earth and Space Sciences, University of California, \\ Los Angeles, CA 90095-1567, USA
}

\begin{abstract}
The dynamics of the jovian magnetosphere are dominated by the mass loading of the lo torus. Sulfur dioxide and possibly other gases such as hydrogen are sputtered from Io and are ionized. These ions in turn are accelerated by the corotational electric field. The hot torus moves slowly outward and then ever more rapidly until reconnection releases the ions down the tail. The outward transport is far from steady. Near Io are intense ion cyclotron waves. As observed by Galileo these waves weakened rapidly on the side of Io closest to Jupiter. Beyond 10 Io radii $\left(R_{\mathrm{Io}}\right)$ at periods less than 150 s there were no discernable waves above the instrument noise level. On the side of Io away from Jupiter the Io cyclotron waves disappeared at about $20 \mathrm{R}_{10}$ and the noise level in the torus reached values almost as low as those inside the Io orbit. However, as the radial distance increased so did the noise levels. The noise levels from 0.01 to $0.1 \mathrm{~Hz}$ are about 2 orders of magnitude greater at $7.7 \mathrm{R}_{\mathrm{J}}$ than inside the Io orbit at $5.5 \mathrm{R}_{\mathrm{J}}$. The noise appears to be due to steps in the magnetic field strength and direction perhaps due to the interchange of tubes of flux with differing plasma content. The transverse power spectral density is greater than the compressional power in this region. The next region in which we have observations is the orbit of Europa and beyond. At frequencies of about $1 \mathrm{~Hz}$ the waves are more strongly compressional than transverse in the equatorial regions and more transverse than compressional off the equator. The outer edge of the dipolar region is about $24 \mathrm{R}_{\mathrm{J}}$. The noise just inside this distance is quite variable from rotation to rotation. Outside this distance in the magnetodisk the field crossing the current sheet varies significantly from orbit to orbit as if the magnetodisk were globally unstable. Beyond about $40 \mathrm{R}_{\mathrm{J}}$ the location of the current sheet oscillates with a period of about 10 minutes. Beyond $50 R_{J}$ the plasma enters a regime of intermittent reconnection. $\odot 2000$ COSPAR. Published by Elsevier Science Ltd. All rights reserved.
\end{abstract}

\section{NTRODUCTION}

Io has long been associated with disturbances in the jovian magnetosphere. Initially it was postulated that Io conducted electric current radially in the jovian magnetosphere, currents that closed in the jovian ionosphere. These currents were in the sense to cause $\mathbf{J x B}$ acceleration of the moon as if the ionosphere was attempting to accelerate lo. The currents flowing parallel to the magnetic field were believed to become unstable, leading to potential drops that caused accelerated electrons and the radio waves that had been observed to be controlled by Io [Bigg, 1964; Goldreich and Lynden-Bell, 1969; Piddington and Drake, 1968]. 
While later papers described the interaction in terms of Alfven waves [e.g. Neubauer, 1980; Southwood, et al 1980] these models retain the basic sense of a unipolar inductor generating a potential drop due to the motion of a conducting Io relative to the magnetized plasma. Pioneer 10 and 11 revealed a magnetodisk-like magnetosphere beyond about $25 \mathrm{R}_{\mathrm{J}}$ [Smith $e t$ al., 1975]. Inside this distance the field was more dipolar. Voyager 1 and 2 were able to provide the density structure in the inner magnetosphere revealing a hot plasma torus from about $10 \mathrm{R}_{\mathrm{J}}$ to $5.9 \mathrm{R}_{\mathrm{J}}$, the orbit of Io [Bagenal, 1985]. The Io interaction was speculated to produce about 1 ton/second of material [Hill et al., 1983]. This material was ionized and quickly accelerated to corotational velocities. As this material slowly drifted outward, currents coupling the magnetospheric plasma to the ionosphere maintained the plasma at close to corotational velocities.

Evidence for the coupling of the magnetospheric plasma to Jupiter's ionosphere comes in the form of swept back magnetic field lines, the twist in the field associated with the field aligned current that provides the ionospheric coupling. Voyager 1 also detected an intense current at Io [Acuna et al., 1981]. This current was limited to the near vicinity of Io and was interpreted to be the unipolar inductor current, albeit now described as Alfven wings closing in a conducting Io. However, the mass added at Io also generates a current system. Thus the Alfven wings may very well be generated, not by a moving conductor in a magnetized plasma, but by mass loading.

In any event the mass added to the jovian magnetosphere makes the magnetosphere a very noisy place. In this paper we explore the activity in the magnetic field from inside the orbit of Io out to $50 R_{J}$. These fluctuations are important because they provide the background fluctuations in which the radiation belt particles find themselves. From the very earliest measurements [Kivelson, 1976] it has been evident that the current sheet in the magnetodisk was much noisier than the lobes on either side and that the compressional power was large in this region. The pre-Galileo observations have been reviewed by Khurana and Kivelson [1989], Khurana [1993] and Glassmeier [1995]. Some of these earlier observations will be recalled as we discuss the Galileo results.

\section{THE INNER MAGNETOSPHERE}

After passing by Io on December 7, 1995 the Galileo spacecraft collected magnetometer data for 30 minutes until $1815 \mathrm{UT}$ at a distance of $5.5 \mathrm{R}_{\mathrm{J}}$. Telemetry was also recorded for 2 hours beginning at 2322 UT at $4.3 R_{\mathrm{J}}$ but no attitude data were recorded so these data are difficult to use. We will attempt to analyze herein only data for which the attitude is well understood. The interaction with Io was extremely dynamic and is described in detail elsewhere [Kivelson et al., 1996; Russell et al., 1997; Huddleston et al., 1997; Warnecke et al., 1997; Russell et al., 1998a]. Ion cyclotron waves were detected beginning about $20 \mathrm{R}_{\mathrm{lo}}$ before Galileo passed through the wake and ceased after $10 \mathrm{R}_{\mathrm{lo}}$. These waves were left-hand polarized, nearly circular, propagating nearly along the magnetic field and oscillating near the $\mathrm{SO}_{2}{ }^{+}$ gyrofrequency. Amplitudes reached over $100 \mathrm{nT}$ peak to peak. The wake itself was populated with mirror mode waves of even larger amplitude. These waves were strongly compressional, and aperiodic in occurrence, with a thickness of several $\mathrm{SO}_{2}{ }^{+}$ion gyroradii. We recall that Pioneer 10 crossed the Io $\mathrm{L}$ shell about $52^{\circ}$ downstream from Io and about $20 \mathrm{R}_{\mathrm{lo}}$ off the magnetic equator. Transverse fluctuations were observed at this location with an rms amplitude of about $10 \mathrm{nT}$ [Walker and Kivelson, 1981]. Thus while the disturbances of the plasma immediately associated with Io have a limited radial extent they do appear to extend far downstream from Io.

Once inside of $5.8 R_{J}$ the field became very quiet. This is illustrated in Figure 1 where the bottom trace shows the transverse power (left) and the compressional power (right). Outside the orbit of Io and beyond the ion cyclotron wave region, the wave level is also low as represented by the 1650 UT spectrum at 6.6 
$R_{\mathrm{J}}$ but not quite as low as inside the Io orbit. Since waves associated with the pitch angle scattering of particles appear to be absent in this region we expect that the plasma is stable with respect to the ion cyclotron resonance. A possible source of free energy for wave generation is the interchange of massloaded flux tubes. If this is true, then in the region near where this spectrum is taken the outward transport of the iogenic plasma is slightly, but not very, unsteady, because there is very little noise in this region.

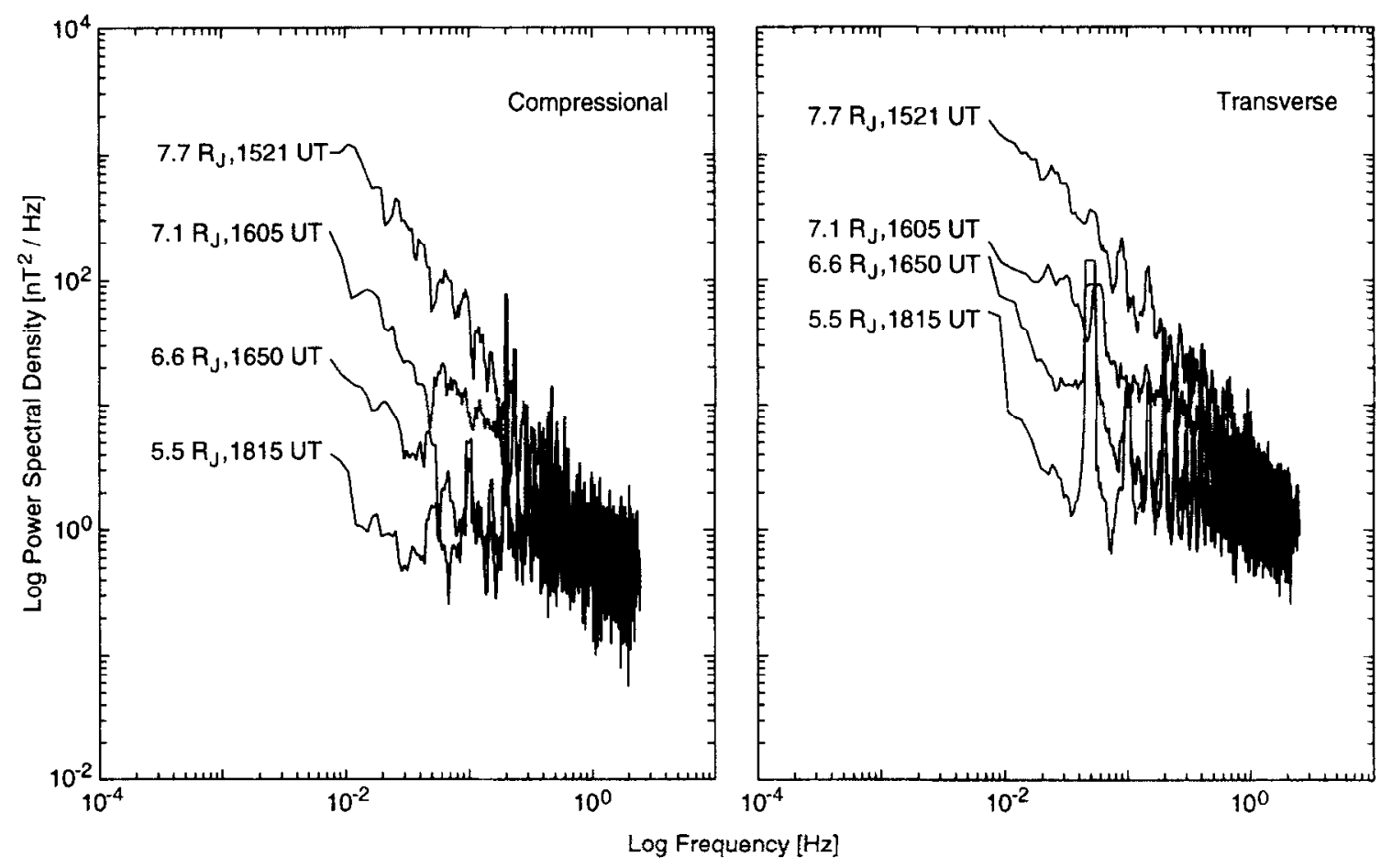

Fig. 1. Power spectral density of the magnetic field obtained on the Io flyby pass on December 7, 1995 at 4 radial distances: $5.5 \mathrm{R}_{\mathrm{J}}$ at $1815 \mathrm{UT}$; $6.6 \mathrm{R}_{\mathrm{J}}$ at $1650 \mathrm{UT}$; $7.1 \mathrm{R}_{\mathrm{J}}$ at $1605 \mathrm{UT}$; and $7.7 \mathrm{R}_{\mathrm{J}}$ at $1521 \mathrm{UT}$. The compressional component is calculated from the magnitude of the magnetic field. The transverse power is the power summed over all three vector components with the compressional power removed. The harmonic structure seen above $50 \mathrm{mHz}$ in the transverse spectrum are due to the incomplete removal of effects associated with the spin of the spacecraft in these data.

The low frequency wave amplitude increases with radial distance. Figure 1 also shows the power spectral density at 1605 UT and a radial distance of $7.1 R_{\mathrm{J}}$. The power continues to increase. At low frequencies the transverse power is about equal to the compressional power. Figure 2 shows the time series corresponding to these power spectra. There is a general increase in the level of activity, but there are also spikes seen principally in the ficld magnitude. We interpret these spikes as empty flux tubes moving inward rapidly after losing their plasma in the reconnection region [Russell et al., 1998b].

The uppermost trace in Figure 1 is the power seen at $1521 \mathrm{UT}$ at a radial distance of $7.7 \mathrm{R}_{\mathrm{J}}$. As before the power is similar parallel and transverse to the field. As the time series in Figure $2 \mathrm{~b}$ shows the magnetic field consists of many step-like disturbances. These disturbances may be due to the interchange instability [Kivelson et al., 1997]. They also may be associated with the fluctuations reported by Glassmeier et al. [1989] in this same region at slightly longer periods. Unfortunately we do not have high resolution data on this orbit at greater radial distances. We must depend on data taken at a rate of one sample every 12 to 24 seconds and distances greater than $10 \mathrm{R}_{\mathrm{J}}$ to complete our journey outward. 

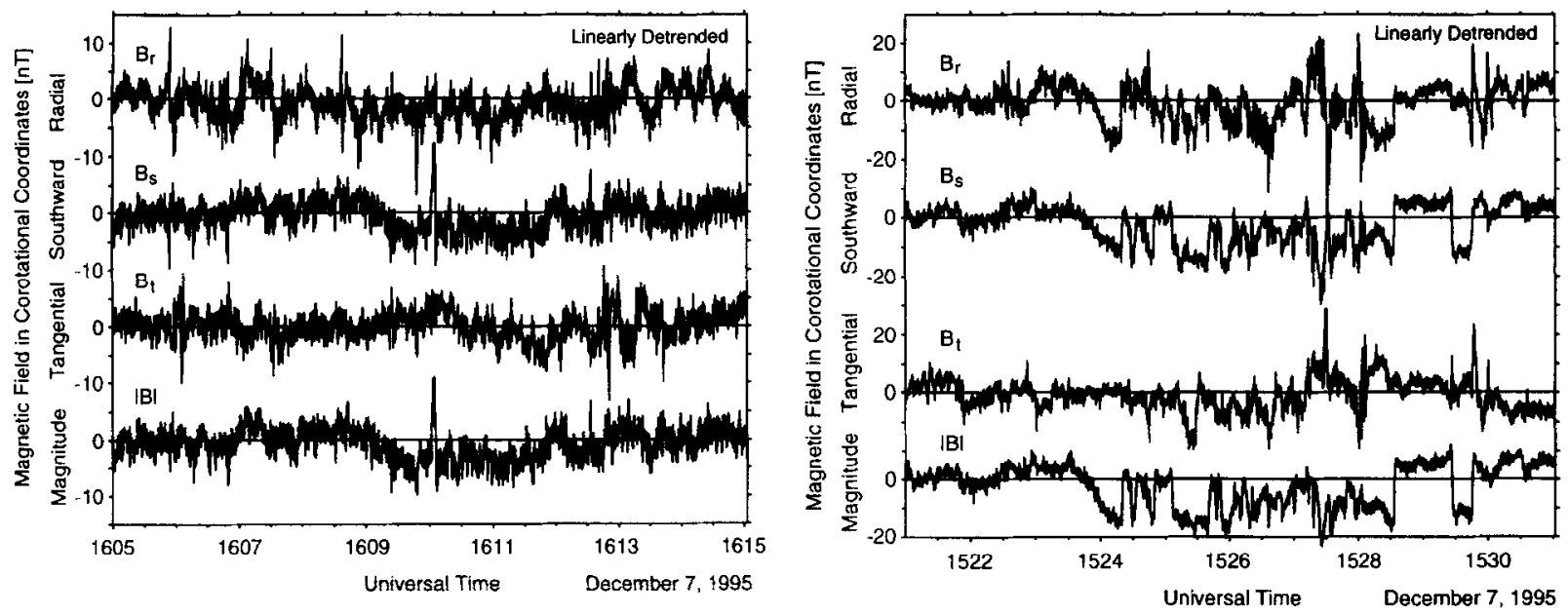

Fig. 2. Linearly detrended time series corresponding to the intervals over which two of the power spectra in Figure 1 were calculated left panel) 1605 - 1615 UT; right panel) 1521-1531 UT.

\section{THE MIDDLE MAGNETOSPHERE}

The jovian magnetosphere from the orbit of Europa to the inner edge of the current sheet at about $24 R_{J}$ is the region we term the middle magnetosphere. A survey of the G2 inbound pass has been reported by Russell et al. [1998c]. We summarize those results herein. We do not discuss the observation of an outward drifting Europa wake or inward drifting empty flux tubes that are seen in this region as they pertain more to the circulation and dynamics of the magnetosphere that are discussed elsewhere [Russell et al., 1998b]. Because of the telemetry limitations of the Galileo mission the data rate in the middle magnetosphere away from the satellite encounters is generally either a sample every 12 or 24 seconds. These are frequencies generally well below the local ion gyrofrequencies.

Figure 3 shows power spectra densities obtained at a radial distance of $10.7 R_{J}$ near and off the equator in the left and right hand panels respectively. The compressional power and the transverse power are shown separately. The compressional power is calculated from fast Fourier transform of the magnitude of the field. The transverse power is the total power summed over the three sensors minus the compressional power.

The compressional power and the transverse power vary with latitude out of phase. The compressional power is strongest near the equator and weakest at high latitudes. The transverse power is greater at high latitudes than at low latitudes. Near the equator the compressional power is greater than the transverse power below about $5 \mathrm{mHz}$ otherwise the transverse power usually dominates in this region. At high latitudes there is little compressional power at any frequency.

Further out in the magnetosphere where the magnetodisk current layer is beginning to form, the fluctuations at the current layer crossing are more isotropic across the entire frequency band so that the compressional and transverse powers are nearly equal and about an order of magnitude greater than the total power seen in Figure 3. This is illustrated with the top spectra of Figure 4 for a current sheet crossing at $23 \mathrm{R}_{\mathrm{J}}$. In contrast in this region off the equator the wave spectrum is very quiet. This is shown with the lower spectra of Figure 4 taken at $25 R_{J}$ where the waves are about a factor of 200 lower in power than spectra at the equator. 


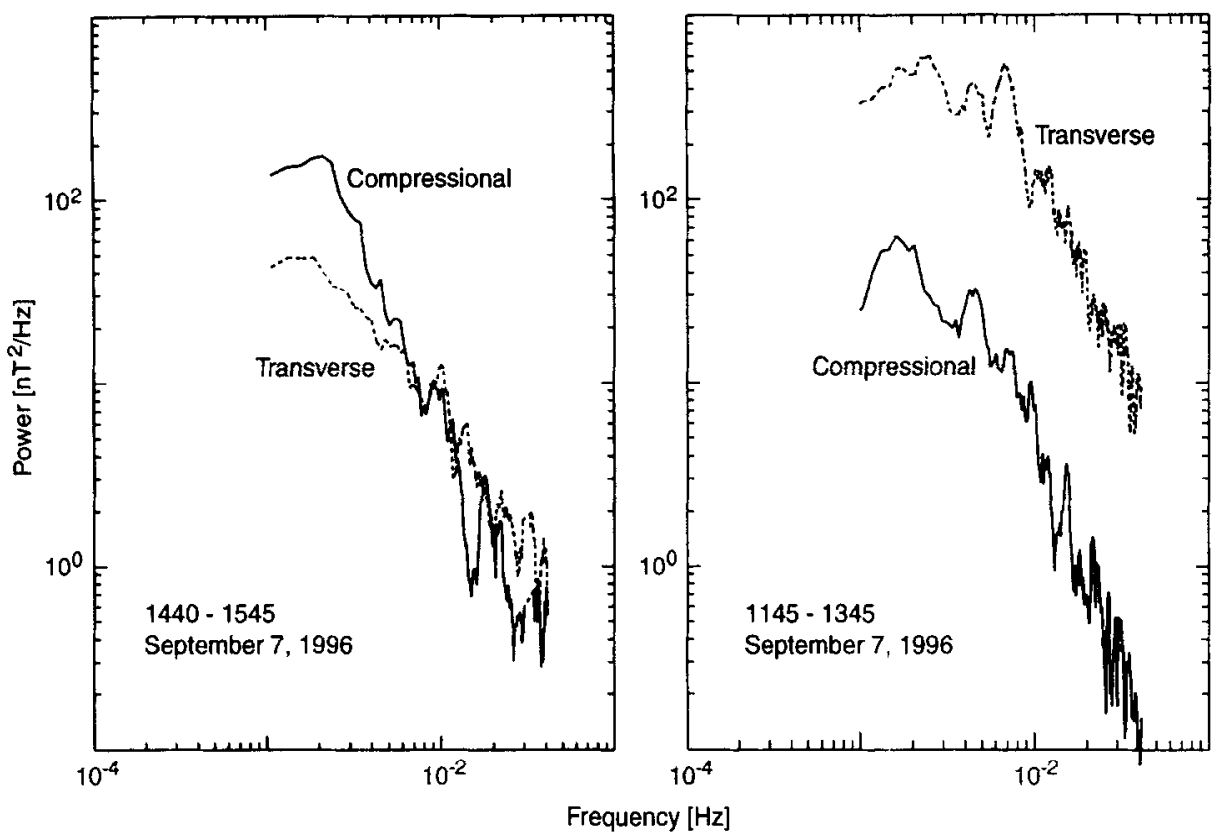

Fig. 3. Power spectral density of the magnetic field seen on the $\mathrm{G} 2$ Galileo pass at a radial distance of $10.7 \mathrm{R}_{\mathrm{J}}$. The compressional and transverse power are shown separately. a) Spectra obtained from 1440-1545 UT on September 7, 1996 near the magnetic equator. b) Spectra obtained from 1145-1345 UT at the highest magnetic latitudes reached by Galileo (Russell et al., 1998c).

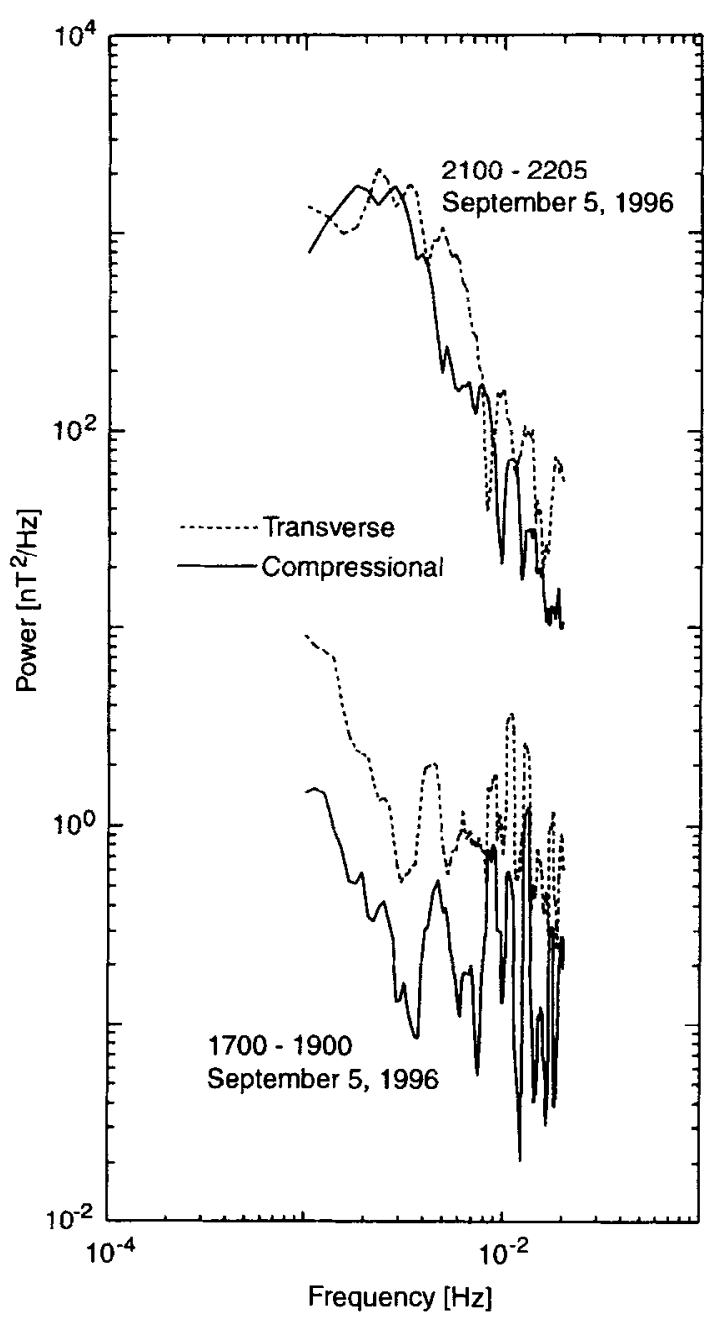

In summary the wave power in the middle magnetosphere is strong but not as strong as in the outer part of the torus. Near the equator the waves are much more strongly compressional than off the equator, and in the outer part of the inner magnetosphere, or more properly above and below the inner part of the magnetodisk even the transverse waves are weak.

\section{TRANSIENT EVENTS IN THE OUTER MIDDLE MAGNETOSPHERE}

On the G2 inbound pass there was a gradual transition of wave properties with radial distance. This was not true on G1. As the spacecraft crossed into the quasi dipolar region of the magnetosphere near $24 \mathrm{R}_{\mathrm{J}}$ and left the magnetodisk the magnetic field became very disturbed for an entire rotation [Russell et al., 1998d]. The time series for this period is shown in Figure 5a where the data have been high pass filtered with a corner frequency of $0.75 \mathrm{mHz}$. The waveforms are quite irregular even spiky in appearance. This disturbance lasted an entire rotation

Fig. 4. Power spectral density of the magnetic fjeld seen at the inner edge of the magnetodisk on the $\mathrm{G} 2$ inbound pass at radial distances of 23 and $25 R_{\mathrm{J}}$. (Top) Measurements crossing the current sheet from 2100 to 2205 UT September 5, 1996. (Bottom) Measurements obtained in the lobes away from the current sheet crossing over the period 1700 to 1900 UT (Russell et al., 1998c). 
and then became much quieter as illustrated in the next rotation of the planet shown in Figure $5 \mathrm{~b}$. Because the disturbance lasted an entire rotation it must have been global in nature. Figure 6 contrasts the noise seen during these two successive rotations beginning at 0830 UT and 2130 UT on June 26, 1996. At lowest frequencies the power is enhanced a factor of 10 in both the transverse and compressional power and a factor of 3 at the highest frequencies. During both intervals, the power falls off more rapidly in the compressional component than the transverse so that at highest frequencies (here $20 \mathrm{mHz}$ ), the waves are mainly transverse.
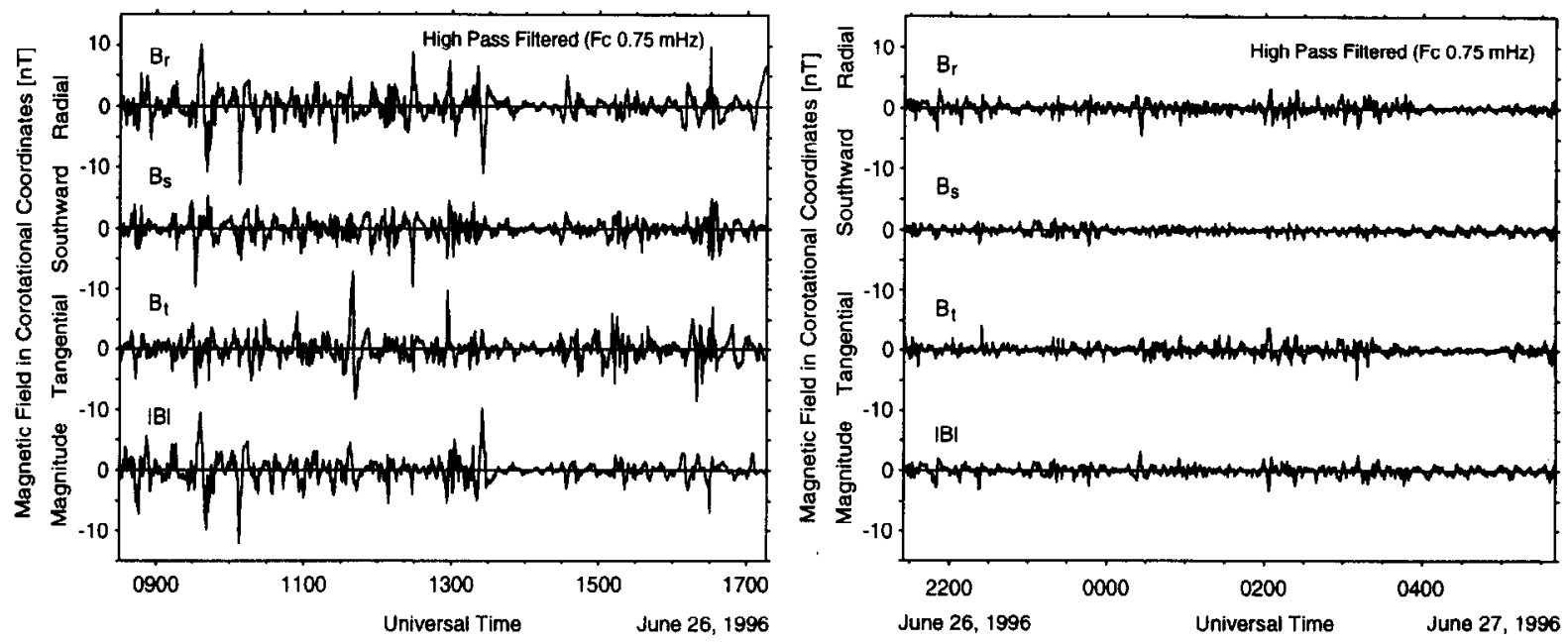

Fig. 5. High pass filtered magnetic field observations in the outer portion of the middle magnetosphere on the G1 inboard pass during a disturbed period. a) the period 0830 to 1715 on June 26,1996 b) the period 2130 of June 26 to 0545 of June 27 , 1996, the jovian rotation immediately following the first interval. The periods are not exactly contiguous because of a data gap.

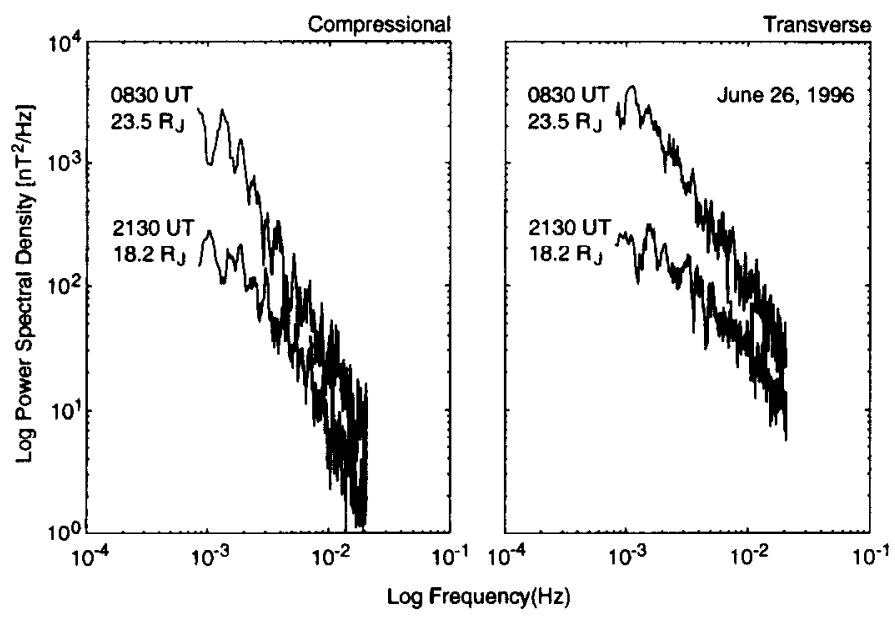

Fig. 6. Compressional and transverse power spectral densities corresponding to the time intervals in Fig. 5.

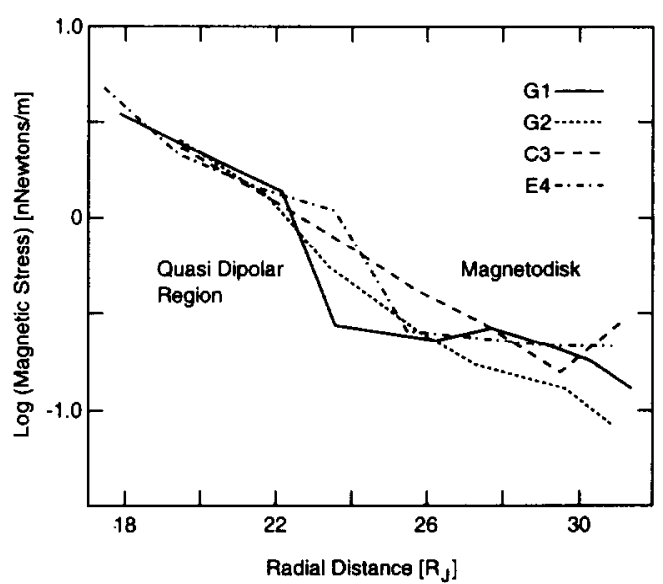

Fig. 7. Magnetic stress integrated across the current sheet for the first 4 inbound Galileo passes as a function of radial distance (Russell et al., 1998d).

\section{THE MAGNETODISK}

The transient disturbance in the outer part of the middle magnetosphere is our first clue that the 
magnetosphere can change from day to day. We also have evidence for this in the variability of the component of the magnetic field that crosses the current sheet. This variability has been discussed by Russell et al. [1998d]. The cross product of this component and the current is a measure of the magnetic force on the plasma, the curvature and pressure forces. We can estimate the current density from the change in the radial component of the magnetic field across the current sheet. The magnetic force as a function of radius, calculated at each current sheet crossing is shown in Figure 7. While the magnetic stress in the middle magnetosphere is relatively constant from pass to pass the stress beyond $24 R_{J}$ in the magnetodisk is quite varied from pass to pass.

We can carry this analysis one step further by estimating the pressure in the current sheet and how it varies with radial distance. Since the three principal forces on the plasma are the magnetic force, the particle pressure force and the centrifugal force, we can estimate the centrifugal force from these other two forces assuming they are in balance. If we assume further that the plasma is rotating with the same period as Jupiter, then we can calculate the mass density from the centrifugal force. This has been done by Russell et al. [1998d] and they find that the radial fall off in plasma density is generally quite smooth except for one of the orbits $\mathrm{G} 2$ when the density appeared to drop to lower levels. This could indicate that there is variation in the density profile or that one of our assumptions is incorrect on this orbit. Since the iogenic heavy ions must be carried radially outward in the magnetosphere, this declining radial density profile can be used to calculate a radial velocity profile. This has been done by Russell et al. [1998b], who find that the velocity rises from a few m/s at lo to about $50 \mathrm{~km} / \mathrm{s}$ at $40 \mathrm{R}_{\mathrm{J}}$.

Two changes occur in the fluctuations associated with the current sheet as one proceeds outward. First, the strength of the "turbulence" in the current sheet increases relative to the strength of the normal component crossing the sheet. In the inner part of the magnetodisk the turbulence is sufficicntly small that it does not result in reversals of the magnetic field component crossing the sheet. However, at about $45 R_{J}$ and beyond this distance the normal component begins to reverse suggesting that there is tearing occurring in the current sheet [Russell et al., 1998e; 1998f]. It is conjectured that the growth of these tearing islands, allowing low density regions above and below the current sheet to become connected, leads to explosive reconnection of the magnetodisk lobes and the formation of magnetized islands of heavy ions that are ejected from the magnetosphere down the tail. We discuss this process briefly in the next section.

A second persistent fluctuation in the magnetodisk is a "10-minute" oscillation in its position. This is in addition to the motion of the warped current sheet in synchronism with the planetary rotation. The amplitude of this 10 minute wave is greatest at largest radial distances resulting in multiple crossings of the current sheet [Russell et al., 1998e]. This wave is possibly the same phenomenon as seen by Voyager 2 in the magnetodisk region and interpreted as a standing Alfven wave [Khurana and Kivelson, 1989].

\section{THE NEAR TAIL REGION AND THE FORMATION OF EMPTY FLUX TUBES}

The magnetospheric magnetic field is ultimately rooted in the planetary interior. The number of field lines exiting the surface of the planet is fixed and on the time scale of the dynamics of the jovian magnetosphere their location is quite fixed as well. Io keeps adding plasma to the magnetosphere and as we have seen above there is much evidence that it moves slowly outward carrying magnetic flux with it. The magnetosphere cannot lose magnetic field lines threading the surface but it must ultimately lose the ions. Thus there must be a mechanism that separates the magnetic field from the ions and allows the ions to escape from Jupiter. Vasyliunas [1983] proposed a circulation model in which a steady state reconnection line formed across the tail that separated the ion laden ends of the field from the less densely populated inner parts of the field lines. The G8 pass of Galileo through this region allows us to determine if this strongly non-linear process occurs. 


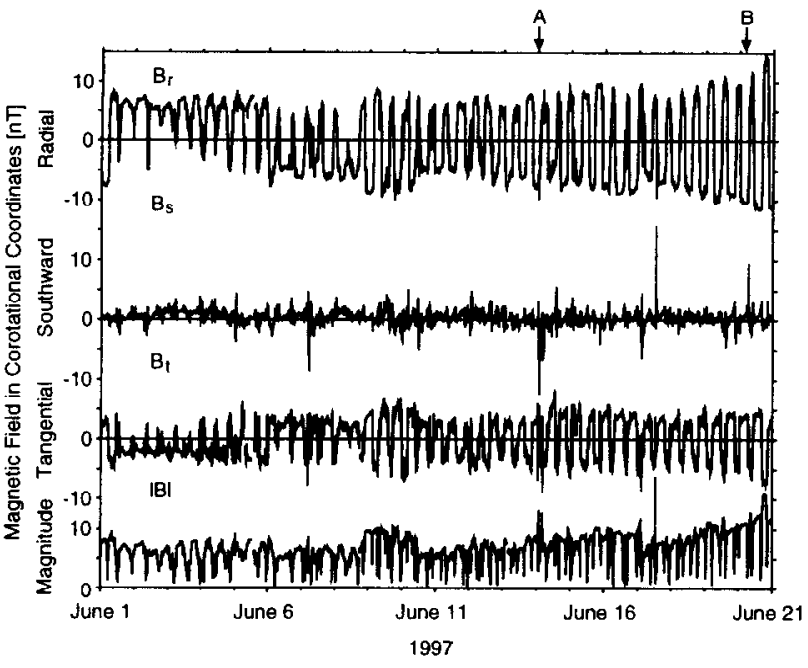

Fig. 8. Magnetic field in the radial, south, tangential (rst) coordinate system as Galileo proceeded from $100 R_{\mathbf{J}}$ to $50 R_{J}$ and from midnight to $0300 \mathrm{LT}$ on the G8 orbit
Figure 8 shows the magnetic field measured by Galileo in the rst coordinate system where $r$ is radially outward, $s$ is southward and $t$ is tangential to the corotational flow direction for the region from $100 \mathrm{R}_{\mathrm{J}}$ to $50 \mathrm{R}_{\mathrm{J}}$ and midnight to $0300 \mathrm{LT}$. The large "sine" wave in $\mathrm{B}_{\mathrm{r}}$ and $\mathrm{B}_{t}$ is the field reversal associated with crossing the magnetotail current sheet. The minor component of the magnetic field $B_{s}$ appears to have a number of "glitches". These glitches are real and last up to an hour in length and represent reconnection, localized in space and time occurring beyond $50 \mathrm{R}_{\mathrm{J}}$ (Russell et al., 1998f,g). The large size of the reconnected normal component indicates the explosive or rapid nature of this process. So while the moon Io provides the ultimate source of energy through the mass loading process, transient reconnection is the process that dynamically releases this energy in the outer magnetosphere.

In addition to leading to a reconfiguration of the magnetic field, the reconnection process creates "empty" buoyant regions whose centrifugal force is much less than that of the unreconnected field. These tubes must interchange with the full field lines to get back to the orbit of Io and repeat the convection cycle. From observations in the middle (Russell et al., 1998c) and inner magnetosphere (Russell et al., 1998b) it appears that the way this process occurs is through the generation of small tubes that can move rapidly through the more slowly moving outward flux. These tubes contain less plasma and hence have higher field strengths than the ion laden flux tubes. Thus they can be detected if the telemetry rate is sufficiently high. To date "empty" tubes have been detected but their size is close to the limits of detectability (Russell et al., 1998b).

\section{SUMMARY AND CONCLUSIONS}

The mass loading at Io sets in motion a large circulation system that begins in the Io torus, transports ions and magnetic flux to the near tail region and then returns the magnetic flux to the inner magnetosphere. This circulation process is unsteady as indicated by the variation seen in the magnetic stresses in the magnetodisk [Russell et al, $1998 \mathrm{~b}$, d]. Inside the orbit of Io the magnetosphere is relatively quiet at frequencies from $5 \mathrm{mHz}$ to $2 \mathrm{~Hz}$. Outside of Io the noise increases with radial distance to at least $7 \mathrm{R}_{\mathrm{J}}$. This noise is in part due to step functions in the field strength that suggest that flux tube interchange is occurring. While the middle magnetosphere is not much noisier than the outer torus magnetosphere, the nature of the fluctuation appears to change with the radial distance beyond the orbit of Europa. Close to the equator the fluctuations are fairly isotropic with roughly equal power in the compressional and transverse waves while at high latitudes the waves are largely transverse to the field. In the magnetodisk region the high latitude magnetosphere is very quiet while the current sheet remains noisy. The strength of this noise increases with radial distance until at about $50 \mathrm{R}_{\mathrm{J}}$ the reversals in the normal component associated with the turbulence become large enough to initiate explosive reconnection across the current sheet. This is not the only "global" instability in the magnetosphere. The properties of the magnetodisk do vary from pass to pass especially the normal component of across the current sheet. On one pass the 
region of the outer middle magnetosphere became disturbed for an entire rotation of the planet. Finally in addition to the motion of the warped current sheet at the planetary rotation period, the current sheet moves up and down with an approximate 10 minute period. It is not clear what causes this fluctuation, dynamics in the inner regions or the outer regions but its amplitude is clearly larger at greater radial distances.

Overall we can ascribe most of the fluctuations in the jovian magnetosphere within about $100 R_{J}$ to endogenic sources. This is not to say that we cannot detect the influences of the solar wind on the system. The solar wind clearly sets the geometry of the tail region. When the solar wind has a northerly flow the tail current is lifted northward above the equatorial plane in which Galileo orbits. When the solar wind dynamic pressure is high, the field strength in the lobes is enhanced. It is possible even that disturbances in the solar wind could trigger disturbances in the outer magnetodisk. However, thus far it is possible to ascribe all the transient disturbances that we see to processes originating within the magnetosphere itself.

\section{ACKNOWLEDGMENTS}

This work was supported by the National Aeronautics and Space Administration through a grant administered by the Jet Propulsion Laboratories.

\section{REFERENCES}

Acuna, M. H., F. M. Neubauer, and N. F. Ness, Standing Alfven wave current system at Io: Voyager 1 observations, J. Geophys. Res., 86 (A10), 8513-8521 (1981).

Bagenal, F., Plasma conditions inside Io's orbit: Voyager measurements, J. Geophys. Res., 90, 311-324 (1985).

Bigg, E. K., Influence of the satellite Io on Jupiter's decametric emission, Nature, 203, 1008-1010 (1964).

Glassmeier, K. H., Ultra low-frequency pulsations: Earth and Jupiter compared, Adv. Space Res., 16(4), 209-218 (1995).

Glassmeier, K-H., N. F. Ness, M. H. Acuna, and F. M. Neubauer, Standing hydromagnetic waves in the Io plasma torus: Voyager 1 observations, J. Geophys. Res., 94, 15063-15076 (1989).

Goldreich, P., and D. Lynden-Bell, Io, a Jovian unipolar inductor, Astrophys. J., 156, 59-78 (1969).

Hill, T. W., A. J. Dessler, and C. K. Goertz, Magnetospheric models, in Physics of the Jovian Magnetosphere, edited by A.J. Dessler, pp. 353-394, Cambridge Univ. Press, New York (1983).

Huddleston, D. E., R. J. Strangeway, J. Warnecke, C. T. Russell, M. G. Kivelson, et al., Ion cyclotron waves in the Io torus during the Galileo encounter: Warm plasma dispersion analysis, Geophys. Res. Lett., 24, 2143-2146 (1997).

Khurana, K. K., ULF waves in other magnetospheres - observations and possible source mechanisms, Ann. Geophys., 11, 973-988 (1993).

Khurana, K. K., and M. G. Kivelson, Ultra low frequency MHD waves in Jupiter's middle magnetosphere, J. Geophys. Res., 94, 5241-5254 (1989).

Kivelson, M. G, Jupiter's distant environment, in Physics of Solar Planetary Environment, (edited by D. J. Williams), 836-853, AGU, Washington, DC, (1976).

Kivelson, M. G., K. K. Khurana, R. J. Walker, J. Warnecke, C. T. Russell, et al., Io's interaction with the plasma Torus: Galileo magnetometer report, Science, 274, 396-398 (1996).

Kivelson, M. G., K. K. Khurana, C. T. Russell, and R. J. Walker, Intermittent short-duration magnetic field anomalies in the Io torus: Evidence for plasma interchange, Geophys. Res. Lett., 24, 2127-2130 (1997).

Neubauer, F. M., Non-linear standing Alfven wave current system at Io: Theory, J. Geophys. Res., 85, 1171-1178 (1980).

Piddington, J. H., and J. F. Drake, Electrodynamic effects of Jupiter's satellite Io, Nature, 217, 935-937 (1968). 
Russell, C. T., F. Bagenal, A. F. Cheng, W.-H. Ip, A. Roux, et al., Io's interaction with the Jovian magnetosphere, EOS Trans. AGU, 78, 93 \& 100 (1997).

Russell, C. T., M. G. Kivelson, K. K. Khurana, and D. E. Huddleston, Magnetic fluctuations close to Io: Ion cyclotron and mirror mode wave properties, Planet. Space Sci., in press, (1998a).

Russell, C. T., M. G. Kivelson, K. K. Khurana, and D. E. Huddleston, Circulation and dynamics in the jovian magnetosphere, Adv. Space Res., submitted, (1998b).

Russell, C. T., D. E. Huddleston, K. K. Khurana, and M. G. Kivelson, The fluctuating field in the middle jovian magnetosphere: Initial Galileo observations, Planet. Space Sci., in press, (1998c).

Russell, C. T., D. E. Huddleston, K. K. Khurana, and M. G. Kivelson, Observations at the inner edge of the jovian current sheet: Evidence for a dynamic magnetosphere, Planet. Space Sci., in press, (1998d)

Russell, C. T., D. E. Huddleston, K. K. Khurana, and M. G. Kivelson, Structure of the magnetodisk current sheet: Initial Galileo observations, Planet. Space Sci., in press, (1998e).

Russell, C. T., K. K. Khurana, M. G. Kivelson, and D. E. Huddleston, Substorms at Jupiter, Adv. Space Res., submitted, (1998f).

Russell, C. T., K. K. Khurana, D. E. Huddleston, and M. G. Kivelson, Localized reconnection in the near Jovian magnetotail, Science, 280, 1061-1064 (1998g).

Smith, E. J., B. V. Connor, and G. J. Foster, Jr., Measuring the magnetic fields of Jupiter, IEEE Trans. Magnetics, 11, 962-980 (1975).

Southwood, D. J., M. G. Kivelson, R. J. Walker, and J. A. Slavin, Io and its plasma environment, J. Geophys. Res., 82, 5959-5968 (1980).

Vasyliunas, V., Plasma distribution and flow in Physics of the Jovian magnetosphere (edited by A.J. Dessler), 395-453, Cambridge University Press, (1983).

Walker, R., and M. G. Kivelson, Multiply reflected standing Alfven waves in the Io torus: Pioneer 10 observations, Geophys. Res. Lett., 8, 1281-1284 (1981).

Warnecke, J., M. G. Kivelson, K. K. Khurana, D. E. Huddleston, and C. T. Russell, Ion cyclotron waves observed at Galileo's Io encounter: Implications for neutral cloud distribution and plasma composition, Geophys. Res. Lett., 24, 2139-2142 (1997). 